\title{
FAST IMAGE ACQUISITION IN MAGNETIC RESONANCE IMAGING BY CHAOTIC COMPRESSED SENSING
}

\author{
Dinh Van Phong ${ }^{\dagger}$, Nguyen Linh-Trung ${ }^{\dagger, *}$, Tran Duc Tan ${ }^{\dagger}, H a V u L e^{\dagger}$, and Minh N. Do $o^{\ddagger}$ \\ ${ }^{\dagger}$ Faculty of Electronics \& Telecommunications, University of Engineering and Technology, VNU-Hanoi, Vietnam \\ ${ }^{\ddagger}$ Department of Electrical and Computer Engineering, University of Illinois at Urbana-Champaign, Illinois, USA
}

\begin{abstract}
We consider the problem of fast acquisition in magnetic resonance imaging (MRI). A recent breakthrough concept called compressed sensing (CS) shows that sparse or, more generally, compressible signals can be recovered from a small number of linear random measurements. CS, using random measurements, has also been successfully applied to MRI for fast acquisition. In a recent work, we have preliminarily employed deterministic chaos in CS that potentially offers a more practical and efficient CS framework. This paper adapts chaotic CS to MRI acquisition. In particular, we use chaotic logistic map for CS and adapt it to acquire the 2-dimensional MRI. In addition, we numerically analyze the performance of the proposed chaotic CS for MRI and show that it performs better random CS.
\end{abstract}

Index Terms - Compressed sensing, MRI, fast acquisition, deterministic chaos.

\section{INTRODUCTION AND STATE-OF-THE-ARTS}

In medical science and technology, Magnetic Resonance Imaging (MRI) has revolutionized diagnostic imaging, thanks to the phenomenon of magnetic resonance of tissue nuclei (e.g., the hydrogen nucleus $\mathrm{H}$ ) present in the object under imaging (e.g., the brain). In principle, by exciting the object with a time-varying excitation RF pulse, the resonance information of the nuclei can be picked up by a receiving RF coil. We take the simple case of acquisition of a full two-dimensional (2D) digital image (e.g., a brain slice) of the object to explain how the image acquisition is done. During a series of RF excitations, in which the 2D locations of points on the brain slice are encoded, the receiving coil receives an analog MRI time signal which contains the resonance information at all encoded locations. The encoded locations are often represented in the so-called $k$-space in which the changes of locations during the acquisition time form a smooth trajectory in the $k$-space. A digital MRI signal is then obtained by sampling the time and the $k$-space. The digital MRI image is then obtained (reconstructed) by applying a reconstruction algorithm on the digital signal to obtain the digital MRI image of the brain slice; for example, we apply the $2 \mathrm{D}$ Fourier transform on the digital MRI signal from the $k$-space to the pixel domain.

Fast image acquisition in MRI is important in order to enhance image contrast and resolution, to avoid physiological effects or scanning time on patients, to overcome physical constraints inherent within the MRI scanner, or to meet timing requirements when

\footnotetext{
*Corresponding author: Nguyen Linh-Trung, nltrung@vnu.edu.vn. This research was supported by VNU-Hanoi under Grant QG.10.40.
}

imaging dynamic structures or processes. State-of-the-art techniques for fast MRI are mainly in the form of parallel imaging in which multiple coils are simultaneously used. Each coil acquires data corresponding to a portion of the imaging object. There exists some redundancy in the acquired data across all the coils. While the acquisition time is inversely proportional to the number of coils, it is this redundancy that can be exploited to reconstruct the final object image. The reconstruction of the image can be done in the image domain, the $k$-space domain or the $k$ - $t$-space domain.

A recent breakthrough in mathematics and signal processing under the name of compressed sensing (CS) shows that sparse or, more generally, compressible signals can be recovered from a small number of linear random measurements [1,2]. Exact reconstruction can be achieved by nonlinear algorithms, using such as $l_{1}$ minimization [1] or Orthogonal Matching Pursuit [3]. In the context of signal sampling, CS is seen as random undersampling. This method is important because many signals of interest, including natural images, diagnostic images, videos, speech and music, are sparse in some appropriate domain of signal representation. Among various applications of CS, it has recently been shown to be successfully applied to MRI for fast acquisition by Lustig, Donoho \& Pauly in [4]. In particular, random undersampling is carried out in the $k$-space. In other words, by acquiring the image with a smaller number of measurements as compared to normal full sampling, the speed of acquisition can be enhanced. Note that if we combine CS and parallel imaging, the speed will be further enhanced. Inspired by this work, further developments in the direction of using CS for MRI continues (see [5] and references therein).

In CS, random measurement process is often used, providing a mathematical convenience for proving exact reconstruction of the signals. This naturally poses a question: can the measurement process be designed deterministically? As an answer to this question, recently, chaotic CS has been proposed in the form of a chaos filter by Linh-Trung et al. [6]. Chaos is aperiodic long-term behavior in a deterministic system that exhibits sensitive dependence on initial conditions. The system is so nonlinear that the output quickly becomes random-like. The proposed chaos filter was numerically showed to perform signal reconstruction better than random filters while enjoying a potential benefit of simple hardware design for chaotic generator as opposed to random generator. In practice, a "random" sequence is generated by a periodic pseudo-random generator, realized by a feedback shift register. A long register is needed to make the period long for the sequence to be more "random", and hence a large storage capacity and logic circuits are needed [7]. In this paper, we apply the method in [6] to MRI for fast acquisition and compare it with the random CS method developed by Lustig et al. [4]. 
The paper is organized as follows. In Section 2, we introduce the principle of compressed sensing theory and MRI acquisition. Section 3 presents our proposed method for MRI acquisition by chaotic measurements. Simulation results to demonstrate the efficiency of our method are presented in Section 4. Section 5 concludes the paper with discussions on the results and remarks for future work.

\section{BACKGROUND}

\subsection{Compressed sensing}

Consider a discrete-time signal $\mathbf{x} \in \mathbb{R}^{N}$ and assume that $\mathbf{x}$ is $K$ sparse in the $N$-dimensional space spanned by the set of $N$ basis vectors $\left\{\Psi_{i}\right\}_{i=1}^{N}$, that is:

$$
\mathbf{x}=\sum_{i=1}^{N} \Psi_{i} s_{i}=\mathbf{\Psi} \mathbf{s}
$$

where $\Psi=\left[\Psi_{1}, \ldots, \Psi_{N}\right]$ is the sparsifying matrix, and $\mathbf{s}=$ $\left[s_{1}, \ldots, s_{N}\right]^{T}$ is the transform vector, containing exactly $K$ nonzero coefficients with $K \ll N$. Examples of commonly used $\boldsymbol{\Psi}$ are Fourier transform, Discrete-Cosine transform, and Wavelet Transform. Note that when $\mathbf{x}$ is a time-sparse signal, i.e. sparse in the time domain, then $\boldsymbol{\Psi}$ becomes the identity matrix. For simplicity of presentation, we restrict ourselves to sparsity, rather than the more general case of compressibility. In the framework of CS, $x$ is linearly acquired by an underdetermined system, represented by a measurement matrix $\boldsymbol{\Phi}$. The obtained measurements $\mathbf{y} \in \mathbb{R}^{M}$, $M<N$, are then given by:

$$
\mathrm{y}=\boldsymbol{\Phi} \mathbf{x}=\Phi \Psi \mathbf{s}=\Theta \mathbf{s}
$$

Given $\mathbf{y}, \boldsymbol{\Phi}$ and $\boldsymbol{\Psi}$, the objective is then to faithfully recover $\mathbf{x}$ (and hence $\mathbf{s}$ ) from $\mathbf{y}$ with the smallest possible value of $M$. If the sparsity information in $\mathbf{x}$ is still fully kept, though being hidden, in $\mathbf{y}$, exact reconstruction of $\mathbf{s}$ is feasible if we find a way to fully restore this sparsity from $\mathbf{y}$. It has been proved that if $\boldsymbol{\Theta}$ satisfies a sufficient condition called the Restricted Isometry Property, then the sparsity information is maintained. A useful indicator for this property is incoherence. $\boldsymbol{\Phi}$ is incoherent with $\boldsymbol{\Psi}$ in the sense that one cannot sparsify the other [8]. One way to ensure the incoherence is to have $\boldsymbol{\Phi}$ as a random matrix with Gaussian i.i.d. elements. Under such a condition, $\mathbf{s}$ can be faithfully recovered from $\mathbf{y}$ when $M$ is such that $c K \log (N / K)<M<N$, where $c$ is some constant, using various sparse approximation techniques, for examples, $l_{1}$-optimization based Basis Pursuit (BP) [1] or Orthogonal Matching Pursuit (OMP) [3].

\subsection{D-MRI acquisition}

Consider the imaging of a 2D slice of the object in the 2D plane $\{x, y\}$. Let $m(x, y)$ be this image. The analog signal acquired by the receiving coil is represented in a temporary image space, called $k$-space, by the following imaging equation:

$$
v\left(k_{x}, k_{y}\right)=\iint m\left(k_{x}, k_{y}\right) e^{-j 2 \pi\left(x k_{x}+y k_{y}\right)} d x d y,
$$

where $k_{x}$ and $k_{y}$ encode the information of location along the $x$ and $y$ directions of the image respectively, and $k=\left\{k_{x}, k_{y}\right\}$ is said to
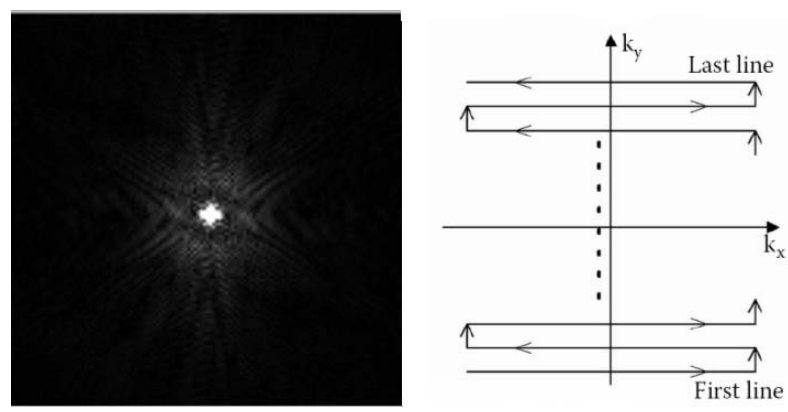

Fig. 1. $k$-space of a brain MR image. Left-full $k$-space showing the concentration of encoded information at the origin. Right-Sampling of the $k$-space along Cartesian trajectory, with linear fullsampling.

lie in the $k$-space. Clearly, the image $m(x, y)$ can be obtained by applying a 2D-Fourier transform on $v\left(k_{x}, k_{y}\right)$. Note that, the time dimension is implicitly included in $k_{x}$ and $k_{y}$. Upon sampling the $k$-space, we have the discrete version of (3) as follows:

$$
v\left(k_{x}, k_{y}\right)=\sum_{n_{x}=0}^{N_{x}-1} \sum_{n_{y}=0}^{N_{y}-1} m\left(n_{x}, n_{y}\right) e^{-j 2 \pi\left(k_{x} n_{x}+k_{y} n_{y}\right)},
$$

where $N_{x}$ and $N_{y}$ are the numbers of pixels along $x$ and $y$ axes of the image. There are various ways of defining $k_{x}$ and $k_{y}$, depending on the $k$-space trajectory in use. A common trajectory for 2D imaging is the Cartesian trajectory used in this paper. Fig. 1 presents the $k$ space of an MR image of a brain slice, which is shown in Fig. 3. We see that most of the encoded information concentrates around the origin of the $k$-space. In practice, the density of the $k$-space follows a power law. We can see from Fig. 1 that most encoded information is concentrated at the origin. In practice, the density of the $k$-space follows a power law.

\subsection{Random CS for MRI using Gaussian random measure- ments}

We have just mentioned that the density of the $k$-space follows a power law. For simplicity, we use Gaussian random measurements for undersampling in the $k$-space. The number of $k$-space samples is much smaller than that by linear fullsampling as described above. MRI reconstruction from the $k$-space samples is performed by Nonlinear Conjugate Gradient (NCG) [4] (see Algorithm 1). Suppose the image of interest is a vector $\mathbf{m}$. The reconstructed image, $\hat{\mathbf{m}}$, is obtained by solving the following constrained optimization problem:

$$
\begin{gathered}
\hat{\mathbf{m}}=\arg \min _{\mathbf{m}}\left\{\left\|F_{u} \mathbf{m}-\mathbf{y}\right\|_{2}^{2}+\lambda\|\boldsymbol{\Psi}\|_{1}\right\} \\
\text { subject to }\left\|F_{u} \mathbf{m}-\mathbf{y}\right\|_{2}<\epsilon,
\end{gathered}
$$

where $\mathbf{y}$ are $k$-space measurements, $F_{u}$ is the undersampled Fourier operator associated with measurements, $\Psi$ is the sparsifying transform operator, and $\lambda$ is a data consistency tuning constant. 


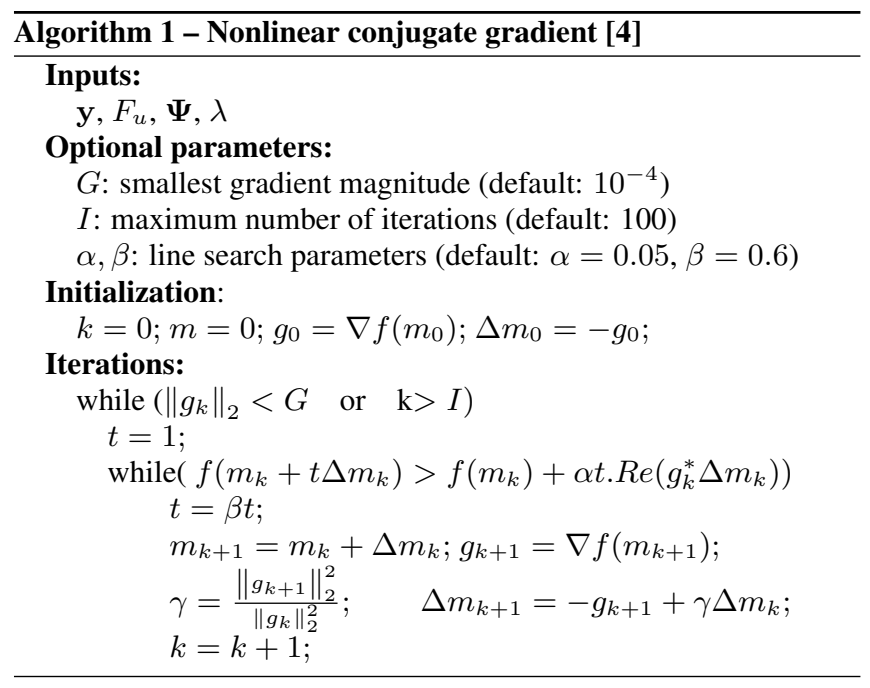

\section{PROPOSED CHAOTIC CS FOR MRI USING LOGISTIC MEASUREMENTS}

In a recent paper [6], we proposed to design the measurement matrix whose elements are generated by chaotic processes rather than random processes. Chaos is aperiodic long-term behavior in a deterministic system that exhibits highly sensitive to initial conditions. The system is so nonlinear that, given appropriate initial conditions, the output quickly becomes random-like. For example, with input $x(n)$, the output of a choatic system called logistic map is given by

$$
x(n+1)=\alpha x(n)[1-x(n)] .
$$

Under suitable conditions (e.g., $\alpha=4, x(0)=0.3), x(n)$ sequence has the chaotic property. Fig. 2(a) illustrates the time-series of the logistic map.

In [6], the chaotic measurements are used to acquire onedimensional sparse signals in the form of a chaos filter. After that, the reconstruction is performed by the OMP algorithm. There, the simulated results indicated that the chaotic approach outperformed the random approach in terms of the probability of exact reconstruction.

In this paper, we use chaotic measurements for MRI acquisition. We generate the values of $k_{x}$ and $k_{y}$ by a logistic map process, and a couple of $k_{x}$ and $k_{y}$ will determine a co-ordinate in $k$-space that will be acquired. However, the distribution of information in $k$-space concentrates nearby the origin and decays when $k_{x}$ and $k_{y}$ increase. Therefore, we convert the distribution of logistic map sequence to Gaussian distribution; its time-series and histogram are shown on Fig. 2(b,c). Once the MRI data have been acquired, the reconstruction is performed by the NCG algorithm. Our scheme can be summarized in Algorithm 2.

\section{SIMULATIONS}

In the simulation, we use a $512 \times 512$ brain MRI for input data, undersampling is performed in the $k$-space by the logistic map. We choose $\alpha=4$ and $x(0)=0.3$. Fig. 3 acquisition of a brain slice (Fig. 3.a) using chaotic CS with compression ratios of 0.15 (Fig. 3.b)

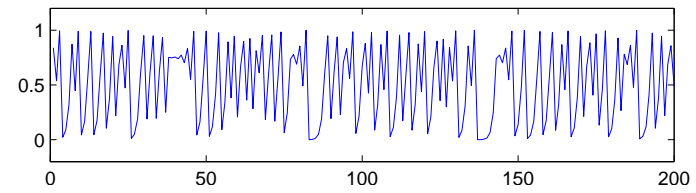

(a) Time-series of logistic map.

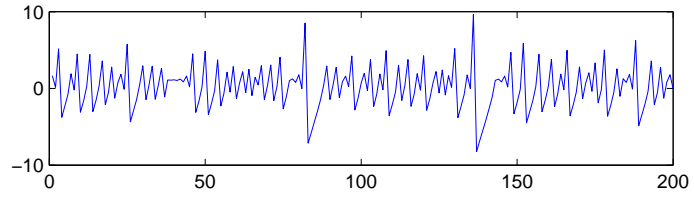

(b) Time-series of Gaussian converted logistic map.



(c) Normalized histogram of Gaussian converted logistic map.

Fig. 2. Logistic map.

and 0.25 (Fig. 3.c). It can be seen that not only the chaotic CS was successful in acquiring the brain image but also, with a compression ratio of around 0.35 , the reconstruction quality was reasonable. Equivalently, the speed of acquisition can be enhanced by around 3 times as compared to using linear fullsampling of the $k$-space.

To compare the efficiency of the design of chaotic measurements, we acquire brain MRI data for a series of compression ratios by measurements which are both chaotic and random. Then, we determine, for each compression ratio, the error in the reconstructed image as compared to the original image. Suppose that $m$ is an $N \times M$ original image and $\hat{m}$ is the reconstructed image. The error can be defined as

$$
e=\frac{1}{N \times M} \sum_{i=1}^{N} \sum_{j=1}^{M}\left|m_{i j}-\hat{m}_{i j}\right|
$$

Fig. 4 shows the results of this comparison. We can see that, for compression ratios that are less than 0.2 , the image reconstructed by chaotic CS has smaller mean-squared-errors than that reconstructed from random CS.

\footnotetext{
Algorithm 2 - Chaos-based CS for MRI acquisition

Step 1: Generate $k_{x}, k_{y}$ that are Gaussian logistic sequences. The number of $k_{x}, k_{y}$ are based on pre-defined compression ratio, $r=$ $M / N$.

Step 2: Determine co-ordinates in k-space based on $k_{x}, k_{y}$ and store as a mask.

Step 3: Acquire digital data in $k$-space based on the mask and store them in a vector $\mathbf{y}$.

Step 4: Reconstruct image by NCG (Algorithm 1).
} 


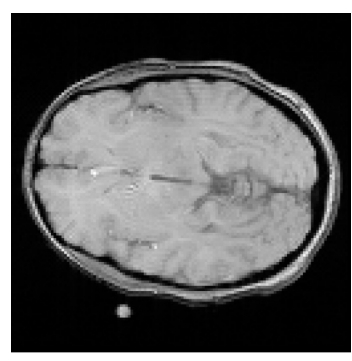

(a) Original brain slice
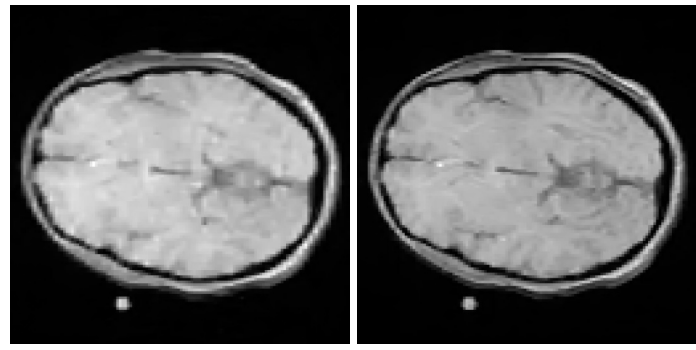

(b) CS with $r=0.15$

(c) CS with $r=0.35$

Fig. 3. Reconstructed images by chaotic CS.

\section{DISCUSSIONS AND CONCLUDING REMARKS}

Based on the work of [6] using chaotic CS for 1D sparse signals, we presented an application to fast acquisition of 2D brain MRI images. Our results confirm the success of using chaotic measurements for MRI acquisition. In addition, chaotic measurements are generated by a deterministic process; hence, we can change and control them by changing initial conditions. This property will be an important advantage when we want to design a hardware system implementing CS for MRI. Lastly, this approach outperforms the random CS proposed by Lustig, Donoho \& Pauly [4] in terms of error performance in image reconstruction.

The reason for better performance of chaotic CS as compared to random CS is currently unknown and, thus, require further investigation to get more insight. In addition, the logistic map is only a simple chaotic process within a large set of processes well developed

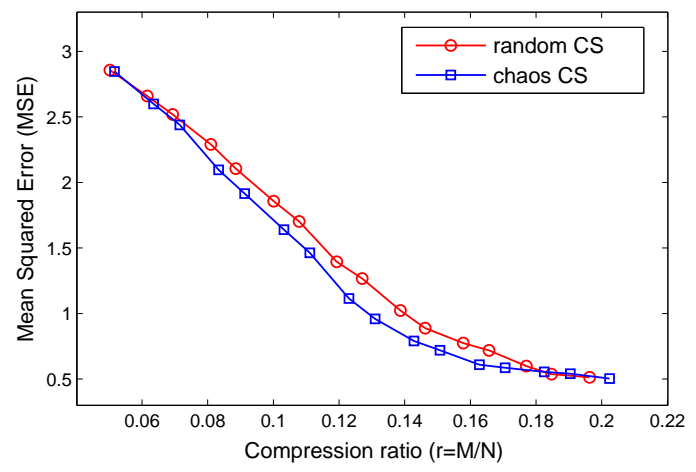

Fig. 4. Performance comparison between chaotic CS and random CS. in chaotic theory. Properties of different chaotic processes and the relationship between these and exact reconstruction conditions in CS should be investigated.

Current CS algorithms are made successful due to the prior information of sparsity, which is inherent in natural signals and images. For examples, MR angiography images viewing blood vessels are sparse in the pixel domain and MRI-scanned brain slices are sparse in the wavelet domain. However, few research results in the context of CS are proposed with additional prior information. These may take the form of hard constraints motivated by the physics of the application, for example the distribution of $k$-space representing the time-varying gradients in an MRI scanner decays by a power law. Another form of prior information is the structural properties of the data, such as angiograms have smooth curves and tree-based representation. Generally, we would be interested in finding out the relationships between structures of chaotic processes and structures of the data.

Interestingly, much development in CS is currently focusing on the theory of CS and the design of efficient algorithms for acquisition and reconstruction of source signals/images based only on one sensor; this can be named as single-channel compressed sensing. An extension to multi-channel CS is not only natural but also practical. In the context of MRI acquisition, due to the need of fast imaging, several well-known parallel imaging techniques have been deployed in state-of-the-art MRI scanners (see [5] and references therein). Our proposed chaotic compressed sensing can be combined with parallel imaging methods to achieve further gain in the speed of MRI data acquisition.

\section{REFERENCES}

[1] Emmanuel Candes, Justin Romberg, and Terence Tao, "Robust uncertainty principles: Exact signal reconstruction from highly incomplete frequency information," IEEE Transactions on Information Theory, vol. 52, pp. 489-509, 2006.

[2] David Donoho, "Compressed sensing," IEEE Transactions on Information Theory, vol. 52, pp. 1289-1306, 2006.

[3] Joel Tropp and Anna Gilbert, "Signal recovery from random measurements via orthogonal matching pursuit," IEEE Transactions on Information Theory, vol. 53, pp. 4655-4666, 2007.

[4] Michael Lustig, David Donoho, and John M. Pauly, "Sparse MRI: The application of compressed sensing for rapid MR imaging," Magnetic Resonance in Medicine, vol. 58, pp. 11821195, 2007.

[5] Hong Jung, Kyunghyun Sung, Krishna S. Nayak, Eung Yeop Kim, and Jong Chul Ye, "k-t FOCUSS: A general compressed sensing framework for high resolution dynamic MRI," Magnetic Resonance in Medicine, vol. 61, pp. 103 - 116, 2009.

[6] Nguyen Linh-Trung, Dinh Van Phong, Z. Hussain, H.T. Huynh, V. Morgan, and J. Gore, "Compressed sensing using chaos filters," in Australian Telecommunication Networks and Applications Conference (ATNAC 2008), 2008, pp. 219-223.

[7] Peter Stavroulakis, Ed., Chaos Applications in Telecommunications, CRC Press.

[8] Emmanuel Candes and Justin Romberg, "Sparsity and incoherence in compressive sampling," Inverse Problems, vol. 23, pp. 969-985, 2007. 